\title{
Designing a Teaching Assistant System for Physical Education Using Web Technology
}

\author{
Shiyuan Qi $\mathbb{D}^{\mathrm{D}}$, Shoubang Li, and Jing Zhang
}

Xi'an Petroleum University, Xi'an 710065, China

Correspondence should be addressed to Shiyuan Qi; shyqi@xsyu.edu.cn

Received 9 April 2021; Revised 10 May 2021; Accepted 29 May 2021; Published 7 June 2021

Academic Editor: Fazlullah Khan

Copyright (c) 2021 Shiyuan Qi et al. This is an open access article distributed under the Creative Commons Attribution License, which permits unrestricted use, distribution, and reproduction in any medium, provided the original work is properly cited.

\begin{abstract}
An efficient and effective management is mandatory for the success of every organization, especially educational institutes. Following the course curriculum and completing it in time are beneficial for the students and the colleges' reputation in educational institutes. In this paper, intelligent management of physical education curriculum is designed using a Web-based teaching assistant system. The multilayer designed system provides convenience for teachers and students of health and physical education. The data layer collects input data from users and transmits it to the storage layer after being compiled by the decoder. The layer uses memory to save the transmitted data and integrate the saved data into the expansion chip and transmit it to the transmission layer. The Web server at this layer outputs the transmitted data in the form of an HTML structure file through a CGI program and feeds back the output to the browser of the application layer. The man-machine interaction interface of the application layer uses a large-scale hierarchical information visualization method to show data on the browser to users. The experimental results show that the system has comprehensive functions, fast processing speed, and low energy consumption. It has a good page display effect, which is in line with the user's visual cognition; the recall rate of curriculum scheduling data and curriculum selection data is always higher than $96 \%$, and the data analysis ability is excellent.
\end{abstract}

\section{Introduction}

In recent years, with the increasing emphasis on students' physical and mental health in colleges and universities, people pay more and more attention to physical education in teaching. Recent researches show that the learning ability of students in a class is uneven and different from others. As a result, in traditional teaching, it is difficult for physical education teachers to take care of the acceptance ability of all students in the centralized classroom teaching. They are often unable to carry out comprehensive teaching according to students' ability, which greatly increases teachers' workload. Besides, the poor communication between students and teachers increases teachers' burden and affects the efficiency of problemsolving. It also makes it difficult to guarantee the learning effect of students, which has a direct impact on the improvement of students' ability and the accumulation of sports knowledge $[1,2]$.
With the rapid increase and widespread applications of computers, multimedia, communication, Web, Internet, and educational technology have entered a new era. Traditional blackboards, tape recorders, slides, and other conventional teaching modes are enhanced by developing multimedia teaching. It is based on computer and network technology, making a qualitative leap in learning methods and educational design [3]. Especially with the rapid development of the Internet, distance-assisted instruction brings the renewal of educational ideas, methods, and means. It provides new ideas and methods for traditional teaching and learning. Nowadays, colleges and universities pay attention to Webbased teaching to expand students' learning ways. As a result, the network-based teaching assistant system has gradually attracted people's attention. Therefore, to improve the teaching quality of the physical education curriculum, the teaching assistant system of the physical education curriculum is designed. The teaching assistant systems integrate the teaching resources, which saves teachers' time. It 
helps students solve complex problems in the learning process of the physical education curriculum. The examination of fundamental theoretical knowledge in the auxiliary teaching system of physical education curriculum can help students consolidate the learning effect of the classroom. Using the communication subsystem can also provide an interactive platform for teachers and students to facilitate teachers' curriculum guidance and improve the efficiency of problem-solving $[4,5]$. The design and implementation of the teaching assistant system change the existing traditional teacher-centered teaching mode to a new student-centered and action-oriented teaching mode.

The design of the teaching assistant system of physical education curriculum is a key research topic, which has attracted many relevant experts and scholars. Yi et al. introduced augmented reality into physical education teaching that improved students' interest in learning by combining a real and three-dimensional virtual model $[6,7]$. Bai et al. introduced students' autonomous learning and teachers' flipped classroom into physical education curriculum teaching. The proposed system was carried out according to the problems and needs faced by students. Then the knowledge construction and ability training were realized with student-centered teaching mode [8]. JohnCrosS et al. used the comparative analysis method to design the system and compared the traditional paper and pen examination with the online examination of the system. They analyzed their respective influences to promote the teaching assistant system of physical education curriculum in colleges and universities. They adopted the constructivism learning theory of Martin Dougiamas, an Australian teacher, and designed the teaching assistant system of physical education curriculum. Web technology has a typical distributed application structure and has gradually developed into the core of network education. This paper designs a teaching assistant system of physical education curriculum based on the Web, which presents the teaching content in the form of page through network transmission. It constructs a network environment to encourage students to participate in physical education learning actively. The system realizes the maximum integration and sharing of physical education teaching resources. It is favorable to promoting the communication between teachers and students and is of great significance to improving the teaching quality of colleges and universities and students' learning quality.

The rest of the paper is organized as follows. In Section 2, the design of teaching assistant systems is elaborated in detail. The detailed results and discussions are given in Section 3, followed by the conclusion in Section 4 .

\section{Design of Web-Enabled Teaching Assistant System for Physical Education}

In this section, we provide details about the subsection of the proposed system.

2.1. Framework of the Proposed System. The design goal of this system is to establish an interactive communication platform for teachers and students, so that teachers can accurately grasp the learning situation of students, so as to improve teaching methods and teaching quality. In the design of the system, the main problem to be solved is how to use the actual teaching workflow to design an intelligent computer program and use the powerful data processing ability of the computer and the flexible and convenient characteristics of the computer network to improve the teaching efficiency [9]. The main contents of the system are online learning, score query, communication, and online examination. The overall framework of the system is described in Figure 1.

2.1.1. Architecture of Online Learning Subsystem. The subsystem comprises three layers: client, Web server, and teaching resource database server, which jointly realize the online teaching and learning of teachers and students. Its structure is shown in Figure 2.

2.1.2. Architecture of Score Query Subsystem. The subsystem comprises a client, WEB server, and score database server, which can meet the needs of teachers and students' score query. Its structure is shown in Figure 3.

\subsubsection{Communication Subsystem. The communication} subsystem can help students get the information they need. It is also a meaningful way to strengthen the communication between teachers and students, and between students and students. Online communication can be divided into two ways: instant discussion and noninstant discussion. Instant discussion requires teachers and students to be online at the same time. This way they can timely discuss problems and get answers. It is an efficient way of learning and communication, but it has certain limitations in time and personnel. The noninstant discussion does not require teachers and students to be online simultaneously. Students and teachers can communicate through SMS, online Q\&A, exchange discussion, and other forms of communication. Although this way is not instant discussion fast, but, not limited by time and personnel, it is very convenient to use [10-13]. Therefore, this system uses noninstant discussion.

2.1.4. Online Examination Subsystem. The subsystem can provide students with the online testing function of curriculum theoretical knowledge. In order to make teachers know students' mastery of the knowledge in time, teachers can set examination questions for students to answer according to the knowledge content of the curriculum. After the students complete the test and submit the test paper successfully, the test paper will be saved in the test papers to be evaluated. After logging into the teaching assistant system of the physical education curriculum, the teachers can view and correct the test paper submitted by the students. After correcting, the students can log into the system to see their total score of the test paper and the detailed information of the test paper, i.e., the score of each paper, correct answers, and the teacher's comments. Through this online test, 


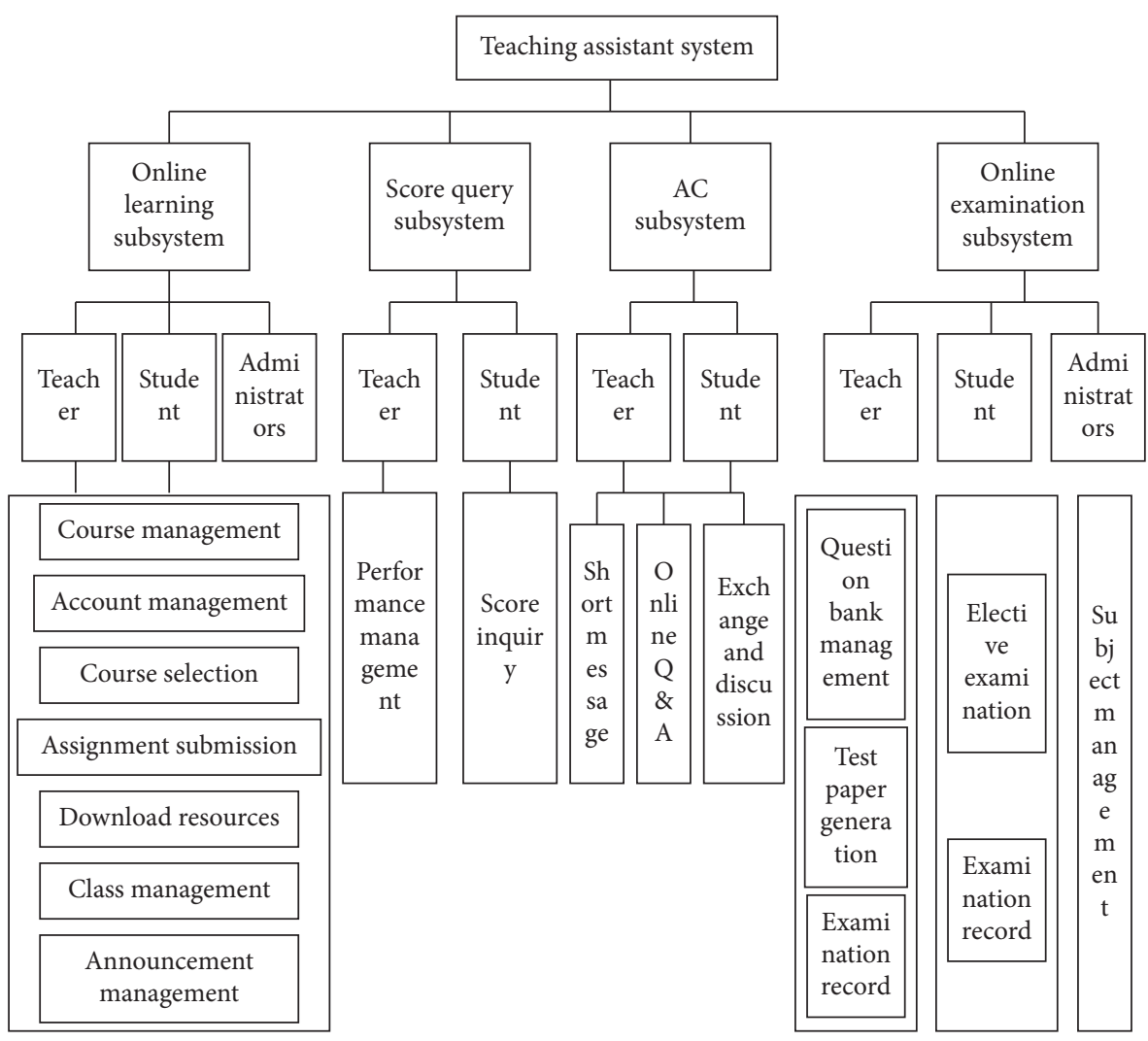

FIgURE 1: System functional architecture.

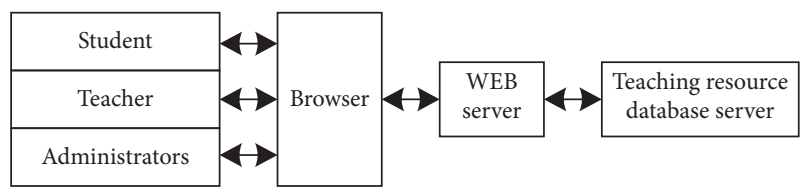

FIgURE 2: Architecture of online learning subsystem.

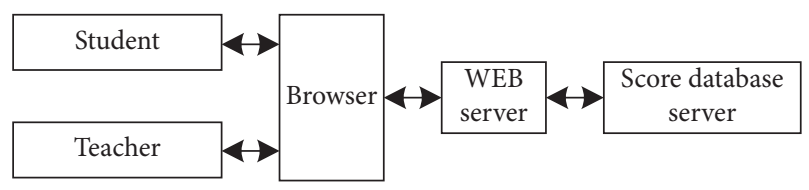

FIgURE 3: The architecture of the score query subsystem.

students can be prompted to consolidate and review to deepen their understanding of knowledge and improve their learning efficiency.

2.2. Structure of System Hardware. In order to apply WEB technology to the teaching assistant system of physical education curriculum and make it play the most influential role, it should have a stable and reliable hardware structure. The details are described in Figure 4.

\subsubsection{Design and CGI Implementation of Embedded WEB} Server. The design and implementation methods are elaborated in this section.

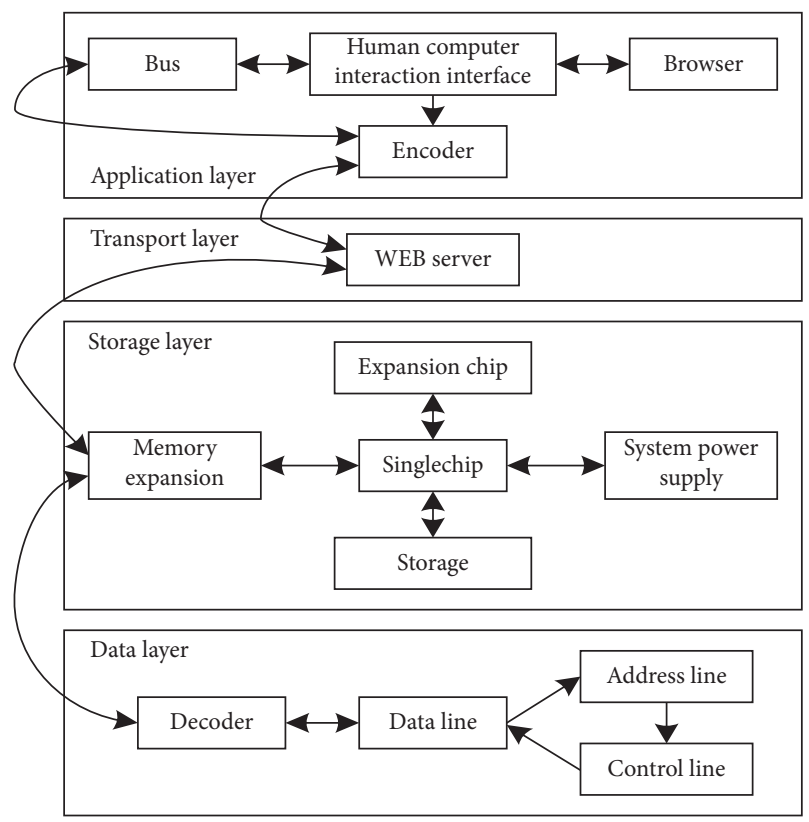

FIgURE 4: Overall structure of system hardware.

(1) Implementation Method in Multitask Environment. A common gateway interface (CGI) can provide a channel to execute external programs in an embedded WEB server. This kind of server technology makes the interaction between browser and server. CGI program belongs to an external program, which needs to be compiled into an executable file 
to run on the server. Its application structure is described in Figure 5. The browser transmits the data input by the user to the WEB server. The WEB server uses Stdin to transmit the data to the CGI program. After the CGI program is executed, it may access some documents storing the data. Finally, Stdout is used to output the structure file in HTML form, transmitted back to the browser through the WEB server and displayed to the user $[14,15]$.

CGI implementation in a multitasking environment is mainly accomplished by HTTPD (such as Apache, uClinux httpd). HTTPD first accepts the HTTP request from the WEB server's analysis and then fork () processes the request. In the subprocess fork (), the corresponding CGI program will be called according to the URL. Before using subprocess to call CGI program, I/O pipeline and CGI environment for the subprocess to communicate with CGI application program are created, and standard I/O of CGI application program is closed. The newly created I/O pipeline description word is converted to the standard $\mathrm{I} / \mathrm{O}$ description word. Then the request is sent to the input pipeline of the CGI application program so that CGI can read and write the pipeline as it operates the standard I/O pipeline.

The advantage of the multitasking CGI implementation method is that it has large throughput. It can process multiple requests in parallel and has a small system overhead, and the size of CGI is easy to control.

(2) Callback CGI Function. Combined with applying the embedded system, considering the need to deal with many user requests and the need to improve the parallel processing speed, reducing the size of the application is very important. In this paper, the specific way to reduce the size of the application is to integrate the traditional CGI application and the embedded Web server module, which can fundamentally reduce the length of the application [16, 17]. In the specific implementation process, the main function (generally the main function) in each traditional CGI application is transformed into a callback function inside the embedded Web server. The embedded Web server is responsible for the callback CGI function after analyzing HTTP requests, as shown in Figure 6.

\subsubsection{Large-Scale Hierarchical Information Visualization} Method. The man-machine interface in the overall hardware structure of the system realizes the interaction between the user and the teaching assistant system of physical education through the large-scale hierarchical information visualization method.

(1) The Radial Drawings Algorithm. The application of the Radial Drawings algorithm can be combined with information visualization technology, hyperbolic browser technology, etc. and ultimately make the layout effect of the teaching assistant system of physical education curriculums more in line with the user's visual cognition. The implementation of the algorithm is very similar to the rooted tree drawing algorithm. Firstly, the root node of the tree structure to be drawn is selected according to the leaf

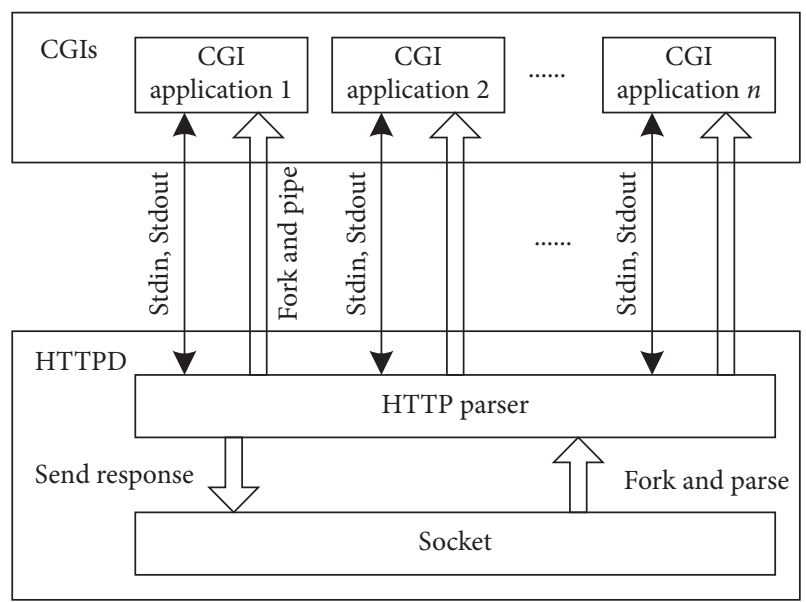

FIGURE 5: CGI application architecture.

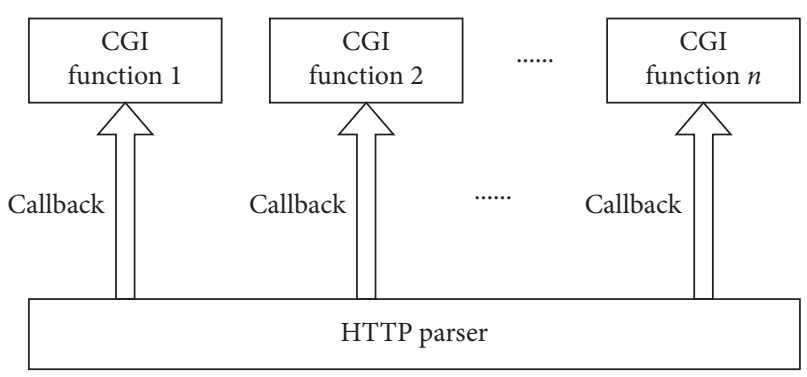

FIGURE 6: Callback CGI function.

pruning algorithm, and the root node is placed in the center of the physical display area. The nonroot nodes are arranged on a series of concentric circles, with the root node as the center. The subtree of the root node is drawn in the wedgeshaped area, and the angle of the wedge-shaped area is determined according to the size of the subtree node. The rendering effect of the algorithm is shown in Figure 7.

(2) Implementation Mechanism of Radial Drawings Algorithm. The core idea of the algorithm is that if $T$ contains leaf nodes, then remove all the leaf nodes in the tree $T$, and then form a new tree by the remaining nodes, which is defined as $T^{\prime}$; perform recursion of this process until the final layout tree does not contain leaf nodes, then define the center of the new tree as the tree center of the layout tree [18].

Assume that the radius of the inner circle of the node with $\mathrm{v}$ as the center is known; assuming that the radius growth of the concentric circle is a fixed value, that is, the value of is a fixed value. In that case, the angle $\alpha$ of the wedgeshaped area can be calculated, expressed as $\tau(\rho)$, and the calculation formula is as follows:

$$
\tau(\rho)=2 * \cos ^{-1}\left(\frac{\rho}{\rho+\delta}\right) .
$$

The Radial Drawings algorithm is implemented recursively. The symbol of the core step of the algorithm is DrawSubtree $\left(v, \rho, \alpha_{1}, \alpha_{2}\right)$ where $v$ represents the root node of the layout subtree and $\rho$ represents the radius of the concentric circle. The node $v$ is located; $\alpha_{1}$ and $\alpha_{2}$ represents the 


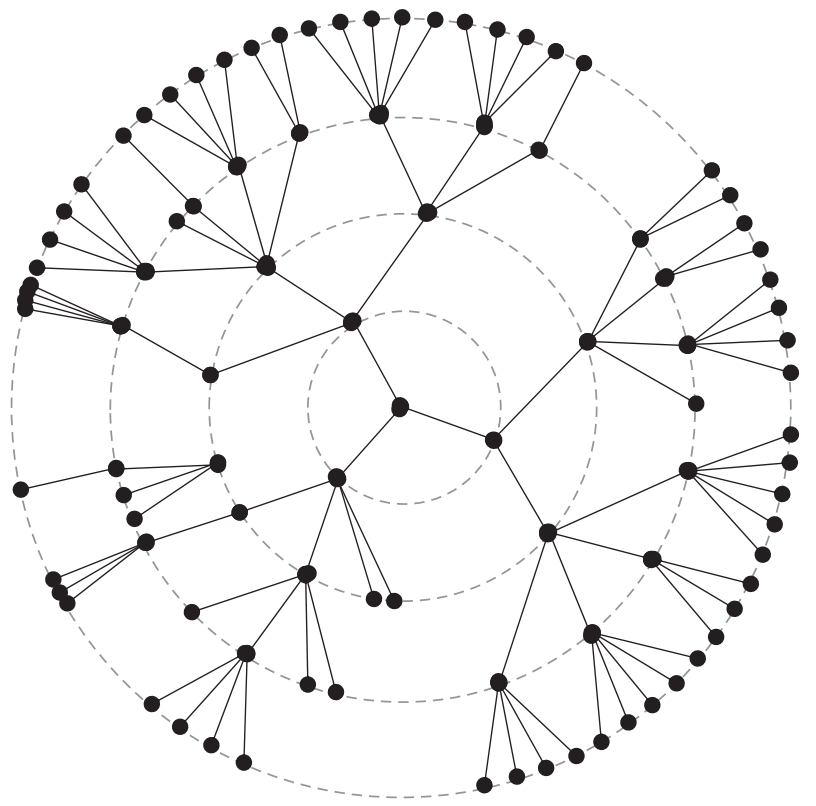

Figure 7: Example of Radial Drawing algorithm.

starting and ending angles of the wedge-shaped area occupied by the subtree of node $v$. Through the corresponding symbol convention, the calculation equation for the angle of the wedge-shaped area of node, and the representation method of recursive program, the detailed flow of the algorithm is obtained, which is described as follows:

Input: random tree $T=(V, E)$;

Output: Radial Drawings layout;

Algorithm: if the center of the tree is a node, call algorithm DrawSubtree $(v, 0,0,2 * \pi)$; if the center of the tree is $d(u, v)$, call algorithms DrawSubtree $(u,(\delta / 2),(3 \pi / 2),(\pi / 2))$,

DrawSubtree $(u,(\delta / 2),(3 \pi / 2),(\pi / 2))$,

DrawSubtree $\left(\nu, \rho, \alpha_{1}, \alpha_{2}\right)$.

(a) Calculate the position of the nodevand express it in polar coordinates.

$$
p=\left(\rho, \frac{\alpha_{1}+\alpha_{2}}{2}\right)
$$

(b) Calculate $\tau(\rho)$, if $\tau(\rho)<\alpha_{2}-\alpha_{1}$, calculate formula (3), and define the initial angle of a subtree, as shown in formula (4):

$$
\begin{aligned}
& \partial=\frac{\tau(\rho)}{w(v)}, \\
& \alpha=\frac{\alpha_{1}+\alpha_{2}-\tau(\rho)}{2} .
\end{aligned}
$$

Otherwise, calculate formula (5) and define the initial angle of subtree as $\alpha \leftarrow \alpha_{1}$.

$$
\partial \leftarrow \frac{\alpha_{2}-\alpha_{1}}{w(v)} .
$$

(c) For each child nodeuof the nodev, recursively call the following algorithm:

$$
\begin{gathered}
\text { DrawSubtree }(u, \rho+\delta, \alpha, \alpha+\partial * w(u)), \\
\partial \leftarrow \alpha+\partial * w(u) .
\end{gathered}
$$

(3) Improved Radial Drawings Algorithm. Radial Drawings algorithm has difficulty in ensuring that the final generated random tree has good uniformity. If there is a volatile situation, it will lead to a tremendous waste of display area. Therefore, to maintain the advantages of the original algorithm and meet the research of large-scale hierarchical information visualization method, we have proposed an unequal space rendering algorithm to improve the performance of the original algorithm.

In the graphics drawn by the algorithm, the equal space characteristic in the ring drawing algorithm is improved so that the space between the concentric circles drawn finally realizes the unequal characteristic. According to the characteristics of the algorithm itself, the unequal spacing characteristics make the algorithm more apparent in the final performance. They can well maintain the user's cognitive bitmap [19, 20]. The improved algorithm adds the interaction mechanism to each node. It adds the scale change mechanism in the program to realize the continuous change of control parameters. It can realize the transformation of image layout according to the expected radius and scale parameters in the layout to choose the focus circle according to their interests. Under the effect of parameters, the overlap rate of nodes in the graph will change with the parameters. Finally, it can minimize the overlap between nodes in information visualization layout rules.

In the improved Radial Drawings algorithm, the nodes in the same depth are still placed on the same ring, but the spacing between rings is no longer equal. It is based on an equal ratio sequence of given points as the variation of ring spacing. Taking the focus ring as the center, the internal and external rings of the focus ring increase and decrease based on the initial radius. The given value of the ring spacing can be calculated during the initial layout, and the proportional value of increase and decrease is limited in the range of $[-1$, 1] [21]. The variables used in the algorithm are defined as follows:

$\rho_{2}$ : The node radius with the expected depth of 2 is used to prevent the radius of the concentric circle with the depth of 2 from being reduced to 0 so that it coincides with the central root node. During the algorithm's execution, the minimum value of the expected radius and the calculated radius are selected as the radius of the concentric circle with a depth of 2 .

$h$ : The variation of loop spacing in interactive navigation.

$s_{r}$ : The scale value of the spacing change in the unequal spacing rendering algorithm, in the range of $[-1,1]$. 
The core idea of the algorithm is that the radius of the concentric circle in the initial layout increases with $h$; that is, the radius of the concentric circle of the node with depth $k$ is $\rho_{k}=(k-1) h$. Assuming the depth of the concentric circle of the focus is $m$, the calculation formula of the radius in the improved algorithm is as follows:

$$
\rho_{k}^{\prime}= \begin{cases}\rho_{m}-\sum_{i=0}^{m-k-1}\left(h^{*}\left(s_{r}\right)^{i}\right), & m>k, \\ \rho_{m}, & m=k, \\ \rho_{m}+\sum_{i=0}^{k-m-1}\left(h^{*}\left(s_{r}\right)^{i}\right), & m<k .\end{cases}
$$

The above formula is defined as when the scaling ratio is greater than 0 . With the focus circle as the center, the change of the radius of the concentric circles on both sides presents a decreasing trend in turn. The change mode is that the radius of the inner concentric circles of the focus circle takes the radius of the focus circle as the reference value and decreases the change of the product of $h$ and scale in turn. The radius of the outer concentric circles takes the radius of the focus circle as the reference value. It increases the change of the product of $h$ and scale in turn. According to the formula analysis, the concentric circles on both sides of the focus spread to both sides.

In formula (8), the radius of the focus circle can be considered, which is not enough to lay out all nodes in the hierarchy under normal circumstances. Suppose that the radius of the drawing origin is $r$, the radius of the focus circle is $\rho_{k}$, and the number of nodes on the focus circle is num; the judgment formula is as follows:

$$
2 * \pi * \rho_{k}>\operatorname{num} * 2 r
$$

A change is selected to the radius of the focus circle. The change is defined as the radius of the focus circle is $R$, and the maximum displayable radius of the physical display area is $\max R$. When the change $h$ and the scale $s_{r}$ are selected, the radius of the focus circle can be expanded according to formula (10). The calculation formula of the change $\Delta \rho_{k}$ is as follows:

$$
\Delta \rho_{k}=\max R-\rho_{k}-\sum_{i=0}^{\text {maxDepth-k }}\left(h^{*}\left(s_{r}\right)^{i}\right) .
$$

The following formula can give the calculation of the value $h$ of the above formula:

$$
h=\frac{\rho_{m}-\rho_{2}}{\sum_{i=0}^{m-2}\left(s_{r}\right)^{i}} .
$$

Formula (8) is modified, with the focus circle as the center, and the ring spacing presents an increasing trend. In this case, it is assumed that the scale provided by the outside is negative, and two arrays are required. Assuming that $A$ and $B$ mark the exponent of the radius change of the inner and outer concentric circles, the definition of the array is as follows:

$$
\begin{aligned}
A & =[m-2, \ldots, 2,1,0], \\
B & =[\operatorname{maxDepth}-m-1, \ldots, 0] .
\end{aligned}
$$

The modification of formula (8) is as follows:

$$
\rho_{k}^{\prime}= \begin{cases}\rho_{m}-\sum_{i=0}^{m-k-1}\left(h^{*}\left(s_{r}\right)^{A[i]}\right), & m>k, \\ \rho_{m}, & m=k, \\ \rho_{m}+\sum_{i=0}^{k-m-1}\left(h^{*}\left(s_{r}\right)^{B[i]}\right), & m<k .\end{cases}
$$

In the above formula, the value of $h$ can be generated dynamically; i.e., the $h$ values on both sides of the focus circle are calculated, respectively:

$$
\begin{aligned}
h_{\text {in }} & =\frac{\rho_{m}-\rho_{2}}{\sum_{i=0}^{m-2}\left(s_{r}\right)^{i}}, \\
h_{\text {out }} & =\frac{\rho_{\text {maxDepth- } \rho_{m}}}{\sum_{i=0}^{\text {maxDepth-m }}\left(s_{r}\right)^{i}} .
\end{aligned}
$$

It can be concluded from formula (14) that when the radius changes in this way, the ring spacing between the concentric rings on both sides of the focus circle shows an increasing trend, which will increase the clarity of the rings near the focus circle. Combining (8) and (14) gives the fact that the two kinds of equation corrections are based on the focus circle as the center to spread to both sides or shrink to the center; that is, the change direction of the radius of the concentric circles on both sides is the same. For the figure modified by formula (14), when the distance between the two sides of the focus circle changes very little, the nodes on both sides will coincide with the focus circle. The changing trend of the radius on both sides can be taken as a phase. In the same direction, the distance between the inner concentric rings increases with the reference of the focus ring. In contrast, the distance between the outer concentric rings decreases $[22,23]$. The formula is modified as follows:

$$
\rho_{k}^{\prime}=\left\{\begin{array}{l}
\rho_{m}-\sum_{i=0}^{m-k-1}\left(h_{\text {in }}{ }^{*}\left(s_{r}\right)^{A[i]}\right) m>k \\
\rho_{m} m=k \\
\rho_{m}+\sum_{i=0}^{k-m-1}\left(h_{\text {out }}^{*}\left(s_{r}\right)^{i}\right) m<k
\end{array}\right.
$$

After the algorithm is modified by formula (17), the nodes on the focus circle will become more apparent in theory. At the same time, due to the overall enlargement of the internal radius, all nodes on the internal concentric circle will become more apparent. The radius change of the outer concentric circle of the focus circle will show a decreasing trend from the focus circle. It can effectively prevent the coincidence of the nodes on both sides of the focus circle and the nodes on the focus circle. 


\section{Results Analysis}

Taking 500 junior students and $20 \mathrm{PE}$ teachers of computer major in a university as the experimental objects, the effectiveness and feasibility of the Web-based PE teaching assistant system are verified. The computer hardware test environment is shown in Table 1.

The experiment tests the function completion of the online learning subsystem and the online examination subsystem and designs a comparative experiment. The engineering graphics mobile teaching assistant system based on the augmented reality technology (referred to as augmented reality technology system) in reference [6] and the hybrid teaching assistant system based on learning behavior analysis (referred to as learning behavior analysis system) in reference [8] are selected as the comparison system of this system. The function completion of the online learning subsystem and online examination subsystem of the three systems is described in Table 2, where success means completed and fail means not completed.

In order to ensure the preciseness and reliability of the test, the test process needs to be simulated many times. According to Table 2, all the functions of the online learning subsystem and the online examination subsystem of the system have been realized. The test results of the four functions of the augmented reality system, i.e., curriculum selection, download resources, test paper generation, and curriculum selection examination, are not completed. It can not meet the needs of users. The test results of six functions of the learning behavior analysis system, i.e., course selection, homework submission, class management, test paper generation, examination selection, and examination record, are incomplete, and the system function integrity is lacking. Comparing these data, we can see that the system's function in this paper is the most comprehensive, which can better achieve the needs of physical education teaching and make the teaching management of physical education more convenient and faster.

The processing speed of the three systems under a different number of users is tested, and the results are described in Figure 8.

The analysis in Figure 8 shows that the processing speed of the three systems increases with the increase of the number of users. However, the processing speed of the proposed system rises very slowly and is always less than $0.4 \mathrm{~s}$. When the number of users is less than 200 , the access speed of the three systems is relatively close. When the number of users is more than 200, the processing speed of the augmented reality system increases rapidly. It then gradually tends to be stable, when the number of users increased to 800 . The processing speed increased to about $1.1 \mathrm{~s}$. The processing speed of learning behavior analysis system continued to rise rapidly; when the number of users increased to 800 , the processing speed reached $1.5 \mathrm{~s}$. Comparing these data, we can see that the system's processing speed in this paper is the fastest, which can provide more efficient services for users.

The overhead of the three systems under a different number of users is tested, and the results are described in Figure 9.
TABle 1: Computer hardware test environment.

\begin{tabular}{lc}
\hline Parameter & Details \\
\hline Graphics card & Intel HD 4000 \\
Processor & Intel core i3 $2.4 \mathrm{GHz}$ \\
Main frequency of processor & $3.20 \mathrm{GHz}$ \\
Memory & $8.00 \mathrm{~GB}$ \\
Memory type & DDR4 \\
Hard disk type & WD10EXEX 500G \\
Hard disk space & Above 20 GB \\
Operating system & Windows 10 \\
Browser version & IE6.0 \\
\hline
\end{tabular}

In this paper, the system's energy consumption is less than $500 \mathrm{~kW} / \mathrm{h}$, and the energy consumption of the system is less than $100 \mathrm{KW} / \mathrm{h}$. However, the energy consumption of augmented reality systems is not affected by the number of users and tends to be stable. Therefore, it can be shown that the proposed system has reduced overhead and better overall performance. Next is the augmented reality system, the learning behavior analysis system has the most significant overhead, and the performance needs to be improved.

The page quality of the three systems under a different number of nodes is tested, and the results are described in Figure 10.

From the analysis of Figure 10, we can see that the resolution of the three systems increases with the increase of the number of nodes. However, this paper's increase is the largest and always at the highest value. When the number of nodes increases to 1000 , the resolution is $700 \mathrm{dpi}$. The resolution and rising trend of the augmented reality system are close to the proposed system. When the number of nodes increases to 1000 , the resolution is $550 \mathrm{dpi}$. The resolution of learning behavior analysis system is relatively low. When the number of nodes increases to 1000 , the resolution is $350 \mathrm{dpi}$. Therefore, it is evident that the page quality of the proposed system is higher, and the page effect is more in line with the user's visual cognition.

Basketball, football, table tennis, synchronized swimming, and other 10 items are set as the subjects of physical education curriculums to test the influence of the system in this paper on the performance of 5 students, and the results are shown in Table 3.

By analyzing Table 3, it can be found that most of the five students' scores in 10 subjects are lower than 90 before using the proposed system. The highest and lowest scores are 94 and 65 , respectively. After using the system in this paper, the scores of the five students in 10 subjects are all above 90 , the score of students' free fight is full, and the scores of all subjects have made great progress compared with before. Therefore, the practical application of the system in this paper can greatly improve the quality of students' learning, and its auxiliary effect is very significant.

The recall rate of curriculum scheduling data and curriculum selection data in this paper is tested. 10 subjects are represented by numbers $1-10$, and the results are described in Figure 11.

The analysis of Figure 11 shows 10 different physical education subjects. The recall rate of the curriculum 
TABle 2: Comparison of subsystem function completion.

\begin{tabular}{lccc}
\hline Function & The paper system & An augmented reality technology system & Learning behavior analysis system \\
\hline Curriculum management & Success & Success & Success \\
Account management & Success & Success & Success \\
Curriculum selection & Success & Fail & Fail \\
Assignment submission & Success & Success & Fail \\
Download resources & Success & Fail & Success \\
Class management & Success & Success & Fail \\
Announcement management & Success & Success & Success \\
Question bank management & Success & Fail & Success \\
Test paper generation & Success & Success & Fail \\
Examination record & Success & Fail & Success \\
Elective examination & Success & Success & Fail \\
Examination record & Success & Success & Fail \\
Subject management & Success & Success \\
\hline
\end{tabular}

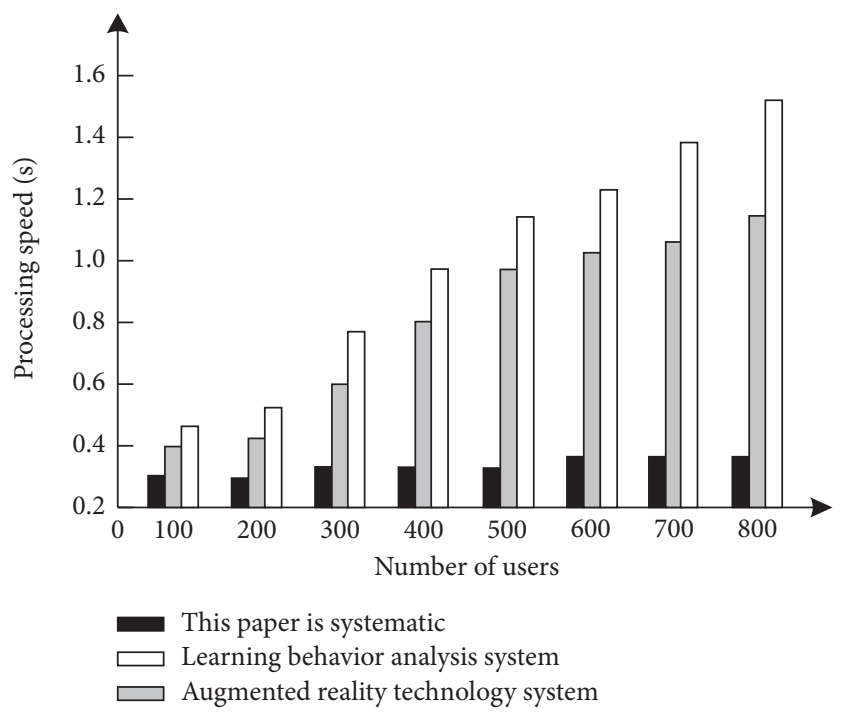

Figure 8: Comparison of processing speed results.

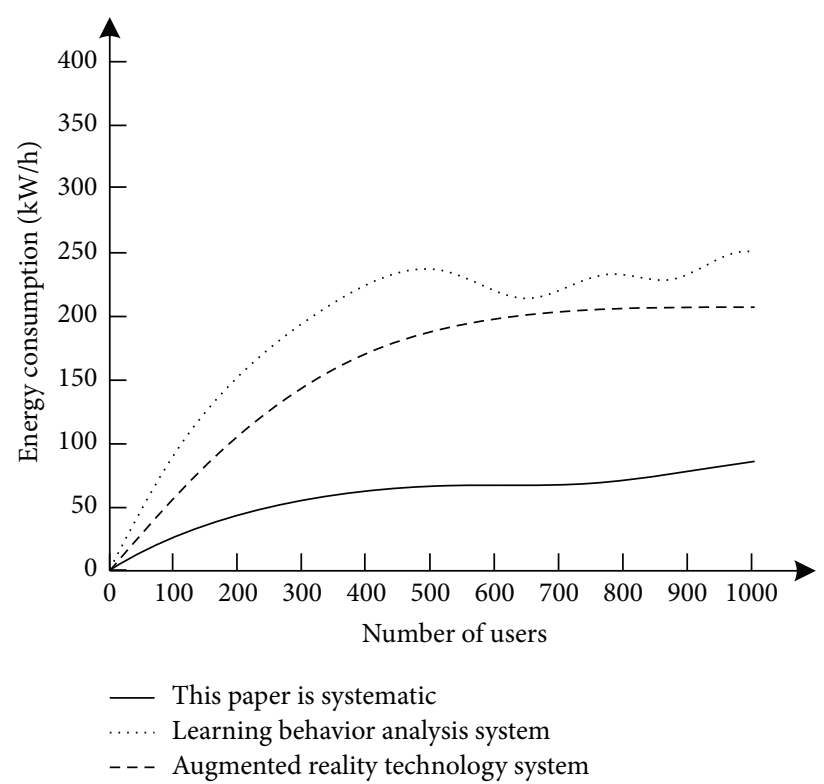

Figure 9: Comparison of system overhead results. 


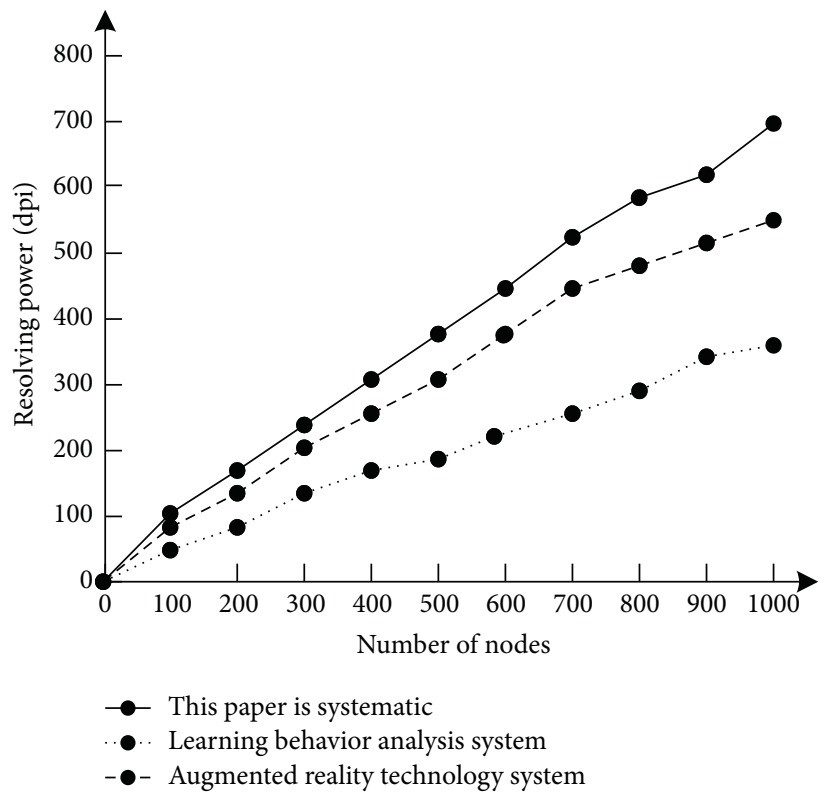

FIgURe 10: Comparison of page quality results.

TABLE 3: Comparison of student achievement results.

\begin{tabular}{|c|c|c|c|c|c|}
\hline Subject & $\begin{array}{l}\text { Student } 1 \text { (unused } \\
\text { grades/used grades) }\end{array}$ & $\begin{array}{l}\text { Student } 2 \text { (unused } \\
\text { grades/used grades) }\end{array}$ & $\begin{array}{l}\text { Student } 3 \text { (unused } \\
\text { grades/used grades) }\end{array}$ & $\begin{array}{l}\text { Student } 4 \text { (unused } \\
\text { grades/used grades) }\end{array}$ & $\begin{array}{l}\text { Student } 5 \text { (unused } \\
\text { grades/used grades) }\end{array}$ \\
\hline Basketball & $85 / 93$ & $91 / 96$ & $68 / 93$ & $67 / 90$ & $65 / 94$ \\
\hline Football & $79 / 90$ & $87 / 93$ & $76 / 88$ & $69 / 91$ & $68 / 91$ \\
\hline Table Tennis & $72 / 91$ & $81 / 90$ & $72 / 90$ & $74 / 95$ & $78 / 92$ \\
\hline $\begin{array}{l}\text { Synchronized } \\
\text { swimming }\end{array}$ & $91 / 95$ & $89 / 98$ & $92 / 95$ & $82 / 94$ & $72 / 96$ \\
\hline Badminton & $84 / 95$ & $90 / 95$ & $87 / 99$ & $81 / 93$ & $89 / 93$ \\
\hline Aerobics & $78 / 92$ & $70 / 92$ & $83 / 92$ & $90 / 95$ & $81 / 98$ \\
\hline Tennis & $80 / 93$ & $69 / 90$ & $80 / 98$ & $88 / 93$ & $87 / 90$ \\
\hline Free fight & $90 / 97$ & $88 / 93$ & $84 / 96$ & $89 / 98$ & $88 / 90$ \\
\hline Taekwondo & $85 / 96$ & $82 / 99$ & $83 / 91$ & $87 / 91$ & $94 / 100$ \\
\hline Taiji boxing & $71 / 90$ & $84 / 96$ & $78 / 91$ & $79 / 86$ & $76 / 93$ \\
\hline
\end{tabular}

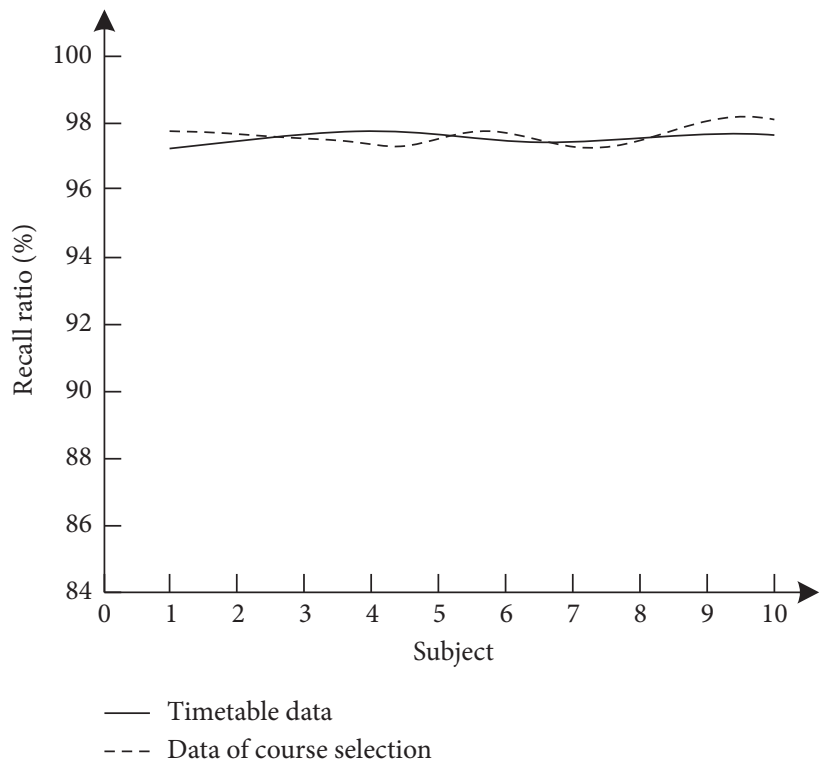

FIGURE 11: Recall ratio of curriculum scheduling data and curriculum selection data. 
scheduling data and curriculum selection data of the system in this paper is higher than $96 \%$, and the highest is $98 \%$. Therefore, it can be shown that the system has good data analysis ability. It can provide a convenient way for teachers and administrators to view the curriculum situation and greatly improve the work efficiency of physical education teachers. Moreover, teachers can also prepare the curriculum content in advance according to the students' curriculum selection situation to improve the teaching quality and make the teaching assistant effect of this system.

\section{Conclusion}

In the information construction of physical education teaching management, we have used modern education concepts and integrated computer networks and multimedia technology. We designed the proposed system based on integrating the above technologies, achieved a more convenient and efficient system, and improved physical education quality. This paper designs a Web-based teaching assistant system of physical education curriculum. The content that needs to be presented is provided to users through the cooperation of the data layer, storage layer, transmission layer, and application layer. Through the experimental analysis, the system has comprehensive functions, processing speed. Energy consumption can reach a better standard, and the page quality is high. The page effect is in line with the user's visual cognition. The practical application of the system can significantly improve the work efficiency and teaching quality of physical education teachers. Compared with the tedious and traditional teaching mode, the vividness, diversity, and comprehensiveness of the system significantly affect students' learning enthusiasm and academic performance. In the future, the system can be further improved to make it widely used in physical education teaching management.

\section{Data Availability}

The data and python code for the simulations are available from the corresponding authors upon request.

\section{Conflicts of Interest}

The authors declare that they have no conflicts of interest.

\section{Acknowledgments}

The authors acknowledge the exploration and practice of "in and out teaching integration" teaching mode in Universities under the background of Internet plus in Shaanxi Province Education Department of Shaanxi Province (17BY047).

\section{References}

[1] A.-P. Correia, N. Koehler, A. Thompson, and G. Phye, "The application of phet simulation to teach gas behavior on the submicroscopic level: secondary school students' perceptions," Research in Science \& Technological Education, vol. 37, no. 2, pp. 193-217, 2019.
[2] Y. Xu, F. Jiang, J. Du, and D. Gong, "A cross-domain collaborative filtering algorithm with expanding user and item features via the latent factor space of auxiliary domains," Pattern Recognition, vol. 94, pp. 96-109, 2019.

[3] M. C. Jung and K. I. Han, "A study on the development of web-based augmentative and alternative communication intervention guide program for teachers," Journal of Emotional \& Behavioral Disorders, vol. 34, no. 4, pp. 289-315, 2018.

[4] J. Qin and L. Wang, "Optimization design of an offshore backfilling plough fenders structure based on the theory of vortex formation," Journal of Coastal Research, vol. 98, no. 1, p. 422, 2019.

[5] Y. Zhang, B. Stadler, R. Henderson, and R. Franklin, "Study of nanowire-based integrated via technology for cmos application in millimeter-wave frequencies," IEEE Microwave and Wireless Components Letters, vol. 99, p. 1, 2021.

[6] P. Yi, Y. C. Liu, Y. J. Shi, Z. Qin, and Z. B. Zhang, "Design and development of engineering graphics teaching system on mobile devices based on the augmented reality technology," Journal of Graphics, vol. 39, no. 06, pp. 195-201, 2018.

[7] Y. Xu, Y. Chu, F. Jiang, Y. Guo, and D. Gong, "SVMs classification based two-side cross domain collaborative filtering by inferring intrinsic user and item features," KnowledgeBased Systems, vol. 141, pp. 80-91, 2018.

[8] L. Bai, Y. L. Hu, and L. Zheng, "A blended teaching model based on learning behavior analysis," Computer Engineering and Science, vol. 40, no. S1, pp. 46-50, 2018.

[9] S.-H. Keng, "Tenure system and its impact on grading leniency, teaching effectiveness and student effort," Empirical Economics, vol. 55, no. 3, pp. 1207-1227, 2018.

[10] A. Woo, A. Rowan, A. Cody, A. Matthew, A. Vance, and B. Matthew, "Senior surgical resident autonomy and teaching assistant cases: a prospective observational study," The American Journal of Surgery, vol. 219, no. 5, pp. 846-850, 2020.

[11] N. Xu and W. H. Fan, "Research on interactive augmented reality teaching system for numerical optimization teaching," Computer Simulation, vol. 37, no. 11, pp. 209-304, 2020.

[12] M. W. Zhao, Z. M. Fan, and R. F. Wang, "Design of synchronous teaching system for hydraulic component theory and experiment based on interconnection," Machine Tool \& Hydraulics, vol. 48, no. 2, pp. 111-118, 2020.

[13] Y. Xu, J. Yang, and Z. Xie, "Training SVMs on a bound vectors set based on Fisher projection," Frontiers of Computer Science, vol. 8, no. 5, pp. 793-806, 2014.

[14] L. J. Yao and L. Zhang, "Design and implementation of chinese architecture history teaching system based on mixed reality technology," Journal of Computer Applications, vol. 39, no. 9, pp. 207-212, 2019.

[15] L. P. Jiang, X. P. Li, L. Zhang, J. Z. Chen, and Y. Y. Dong, "Design of virtual teaching experience space system based on wearable human body perception," China Educational Technology, vol. 407, no. 12, pp. 34-67, 2020.

[16] W. Gong, L. Tong, W. Huang, and S. Wang, "The optimization of intelligent long-distance multimedia sports teaching system for iot," Cognitive Systems Research, vol. 52, no. 1, pp. 678-684, 2018.

[17] X. Yu, D. Zhan, L. Liu, H. Lv, L. Xu, and J. Du, "A privacypreserving cross-domain healthcare wearables recommendation algorithm based on domain-dependent and domainindependent feature fusion," IEEE Journal of Biomedical and Health Informatics, vol. 99, p. 1, 2021. 
[18] K. Hoad and M. Kunc, "Teaching system dynamics and discrete event simulation together: a case study," Journal of the Operational Research Society, vol. 69, no. 4, pp. 517-527, 2018.

[19] R. Mehren, A. Rempfler, J. Buchholz, J. Hartig, and E. M. Ulrich-Riedhammer, "System competence modelling: theoretical foundation and empirical validation of a model involving natural, social and human-environment systems," Journal of Research in Science Teaching, vol. 55, no. 5, pp. 685-711, 2018.

[20] P.-H. Liu and S.-B. He, "Analysis of teaching reform mode based on cognitive computing system - an example of dragon boat teaching," Cognitive Systems Research, vol. 52, no. 1, pp. 978-984, 2018.

[21] W. Ho and K. Tai, "Doing expertise multilingually and multimodally in online English teaching videos," System, vol. 94, no. 3, Article ID 102340, 2020

[22] M. Yu, T. Quan, Q. Peng, X. Yu, and L. Liu, "A model-based collaborate filtering algorithm based on stacked auto encoder," Neural Computing and Applications, vol. 4, 2021.

[23] M.-Á. Serrano, E. Vidal-Abarca, and A. Ferrer, "Teaching selfregulation strategies via an intelligent tutoring system (tuinlec web): effects for low-skilled comprehenders," Journal of Computer Assisted Learning, vol. 34, no. 5, pp. 515-525, 2018. 